\title{
Condições sociais e clínicas associadas à promoção de saúde em pacientes com fissuras labiopalatais atendidos em um centro de referência
}

\author{
Sarah Lindsen Souza Corsini ${ }^{a}$, Larissa Fátima Carvalho ${ }^{a}$, Lorena Carvalho Araújo ${ }^{a}$, \\ Karolinne Becker Moreira ${ }^{a}$, Alessandra dos Santos Danziger Silvério ${ }^{a}$, Gérsika \\ Bitencourt Santos $^{\mathrm{a}}$, Cláudio Daniel Cerdeira ${ }^{\mathrm{b} *}$, Asafe Costa Lopes ${ }^{\mathrm{c}}$
}

${ }^{\text {a} F a c u l d a d e ~ d e ~ M e d i c i n a, ~ U n i v e r s i d a d e ~ J o s e ́ ~ d o ~ R o s a ́ r i o ~ V e l l a n o ~-~ U N I F E N A S, ~ A l f e n a s, ~ M G, ~ B r a s i l ~}$

bDepartamento de Bioquímica (DBq), Instituto de Ciências Biomédicas, Universidade Federal de Alfenas (UNIFAL-

MG), Alfenas, MG, Brasil

'Instituto de Ciências Biomédicas, UNIFAL-MG, Alfenas, MG, Brasil

Histórico do Artigo: Recebido em: 19/05/2020

Aceito em:

$21 / 08 / 2020$

Palavras-chave:

Fissuras

labiopalatais; fatores de risco; promoção de saúde

Keywords:

Cleft lip and palate; risk factors; health promotion

\begin{abstract}
RESUMO
Analisou-se através de prontuários e questionários semiestruturados os fatores sociodemográficos e clínicos que interferem na promoção de saúde de pacientes com fissuras labiopalatais (FLP), atendidos em um centro de referência no Sul de Minas Gerais, Brasil, entre 2016 e 2017. Entre os 202 pacientes, com idade de 1 a 10 anos, houve predominância do sexo masculino $(62,38 \%)$, declarados leucoderma $(82,67 \%)$ e pertencente a classe socioeconômica C $(67,32 \%)$. O grupo sanguíneo O positivo foi o mais frequente. Quanto ao tipo de FLP, as pós-forame incompleto, préforame incompleto e transforame completa esquerda foram as mais comuns, e os tratamentos cirúrgicos mais frequentes foram a queiloplastia e a palatoplastia. Acompanhamento com fonoaudiólogo foi relatado pela maioria dos entrevistados. Quanto aos familiares, 70,8\% das mães fizeram suplementação com ácido fólico durante a gestação e, 55,44\% dos pacientes com FLP tiveram peso ao nascimento entre 3.000 e 4.000 gramas. Por outro lado, foi relatado em considerável frequência $(30,7 \%)$ um histórico de familiares com alguma alteração craniofacial congênita, além de exposição ocupacional a teratógenos por uma parcela das mães. Quanto as comorbidades, as doenças respiratórias, alergias e anemia ocorreram em maior frequência. A frequência de pacientes com histórico familiar para alterações congênitas, associados à uma exposição ocupacional das mães, bem como as classes econômicas menos favorecidas sendo as mais suscetíveis à doença, são fatores que reforçam a plausível etiologia multifatorial das FLP. Ainda, fatores sociais e clínicos interferem na promoção de saúde dos pacientes com FLP.
\end{abstract}

Social and clinical conditions associated with health promotion in patients with cleft lip and palate attended at a referral center

\section{ABSTRACT}

Sociodemographic and clinical factors that interfere in the health promotion of patients with cleft lip and palate (CLP) treated at a referral center in southern Minas Gerais, Brazil, were analyzed through medical records and semi-structured questionnaires between 2016 and 2017. Among the 202 patients, aged 1 to 10 years old, there was a predominance of males $(62.38 \%)$, declared leukoderma (82.67\%) and belonging to socioeconomic class C (67.32\%). The positive O blood group was the most frequent. As for the type of CLP, incomplete post-foramen, incomplete preforamen and complete left transforamus were the most common, and the most frequent surgical treatments were cheiloplasty and palatoplasty. Follow-up with a speech therapist was reported by most respondents. As for family members, $70.8 \%$ of mothers did supplementation with folic acid during pregnancy and $55.44 \%$ of those patients with CLP had birth weight between 3,000 and 4,000 grams. On the other hand, a history of relatives with some congenital craniofacial alteration was reported, with considerable frequency (30.7\%), in addition to occupational exposure to teratogens by a determined number of mothers. As for comorbidities, respiratory diseases, allergies, and anemia occurred more frequently. The frequency of patients with family history for congenital alterations, associated with mothers' occupational exposure, as well as the less favored economic classes being the most susceptible to this disease, reinforce the plausible multifactorial etiology of CLP. Moreover, the social and clinical factors interfere in health promotion of patients with CLP.

\footnotetext{
*Autor correspondente: daniel.cerdeira.84@gmail.com (Cerdeira C.D.)
} 


\section{Introdução}

As alterações congênitas afetam, mundialmente, cerca de 5\% dos nascidos vivos. Entre estas, as alterações orofaciais representam um grupo heterogêneo e complexo $(1,2)$. Aproximadamente $70 \%$ das alterações orofaciais são não sindrômicas e, nesta classe, a fissura labial com ou sem fenda palatina está entre as três mais prevalentes. As fissuras labiopalatais/labiopalatinas (FLP) caracteriza-se por uma abertura ou ruptura na região do lábio e/ou palato, ocasionada pelo não fechamento dessas estruturas durante o desenvolvimento embrionário e/ou fetal humano $(2,3)$.

Apesar da etiologia das FLP não estar totalmente elucidada, evidências demonstram um envolvimento de fatores genéticos (hereditários, dois ou mais genes alterados) e ambientais, portanto, trata-se de uma doença Genética multifatorial, com uma Herdabilidade estimada em torno de $76 \%(3,4)$. A incidência mundial das FLP varia entre 1 em 500-2.500 nascidos vivos e, no Brasil, país que apresenta tendências crescentes, em 2017, foi reportado em torno de 0,6 casos para cada mil nascidos vivos.

Entretanto, a incidência para as FLP pode variar de acordo com as regiões geográficas, grupos étnicos/raciais, sexo, história familiar de FLP, classificação socioeconômica e faixas etárias materna e paterna $(2,3,5)$. Assim, os hábitos deletérios entre gestantes podem ser considerados sérios fatores de risco para o desenvolvimento destas alterações congênitas, e estes incluem tabagismo, uso de álcool ou exposição a outros agentes teratogênicos (tais como agrotóxicos/herbicidas) até o $3^{\circ}$ mês de gestação, além da deficiência nutricional de ácido fólico $(3,5)$.

As FLP podem afetar consideravelmente o palato e o posicionamento dentário, ocorrendo maior dificuldade na capacidade de fala e alimentação, com comprometimento nutricional, bem como o risco de aspiração dos alimentos, provocando infecções, tais como otites e pneumonias. Assim sendo, na ausência de tratamento adequado, as FLP podem provocar sequelas graves, como a perda da audição, problemas de fala e déficit nutricional, além do sofrimento com o preconceito. Por comprometerem estas funções orgânicas básicas, a saúde geral e a estética facial, bem como o desenvolvimento psicológico e adaptação social, além do acompanhamento médico, é necessário a participação efetiva de uma equipe multiprofissional para a promoção de saúde das pessoas vivendo com FLP (6,7). Neste contexto, este estudo analisou as condições sociais e clínicas que interferem na promoção de saúde de pacientes com FLP atendidos em um centro de referência.

\section{Métodos}

Trata-se de um estudo envolvendo 202 pacientes com FLP, voluntários, com idade entre 1 a 10 anos, assistidos no Centro Pró-Sorriso da Universidade José do Rosário Vellano - UNIFENAS, uma clínica universitária especializada em tratar pacientes com deformidades craniofaciais e com lesões/fissuras labiopalatais, conveniada com o Sistema Único de Saúde (SUS), e localizada na cidade de Alfenas, município do estado de Minas Gerais, Brasil. Como critérios de inclusão foram considerados todos os pacientes com FLP, com idade entre 1 a 10 anos, assistidos no referido centro de referência, cujo responsável legal, após esclarecimentos, concordou em participar do estudo e apresentava capacidade de responder aos questionários, bem como o prontuário encontrava-se completo com finalidade de atender as questões do estudo. Como critérios de exclusão, não foram considerados aqueles que se recusaram em participar do estudo e/ou pacientes com prontuários/documentos que apresentavam qualquer um dos dados aqui levantados ausentes. 
Entre novembro de 2016 a dezembro de 2017, após a consulta de rotina agendada, foi realizada à entrevista, sendo que as respostas foram anotadas em um questionário semiestruturado, elaborado pelos pesquisadores, e aplicados em área restrita. As entrevistas foram realizadas por pesquisadores previamente calibrados, ressaltando-se a presença do viés de memória dos entrevistados e o período recordatório para as questões foi dependente dos eventos questionados. A calibração ocorreu em um estudo piloto envolvendo $10 \%$ da amostra final, e o grau de concordância entre os entrevistadores foi maior que $80 \%$ (estatística kappa > 0,8).

As variáveis sociodemográficas coletadas foram: idade, sexo, raça, classe social e tipo de trabalho pela classificação da Associação Nacional de Empresas de Pesquisa (ANEP). Dados referentes à anamnese e as variáveis clínicas, tais como, tipo sanguíneo, o tipo de FLP, comorbidades, peso ao nascimento (em gramas) e história familiar de alterações craniofaciais congênitas em geral (em familiares de primeiro grau: mãe e/ou pai e/ou irmã e/ou filha), e o número de procedimentos cirúrgicos realizados foram transcritos a partir do prontuário do paciente para um formulário específico para este estudo. As FLP foram classificadas de acordo com os critérios de Spina (8), baseada no forame incisivo do palato: Grupo I - fissuras pré forame (lábio e labio-gengival), Grupo II - fissuras transforame (labiopalatal), Grupo III - fissuras pós-forame (palatal) e Grupo IV - fissuras raras da face. Os resultados foram apresentados em valores absolutos e em porcentagem (\%), e um intervalo de confiança de 95\% (IC95\%) foi estimado usando o software BioEstat ${ }^{\circledR} 5.0$ (Belém, Pará, Brasil, 2007).

O presente estudo foi aprovado pelo Comitê de Ética em Pesquisa Envolvendo Seres Humanos da Universidade José do Rosário Vellano - UNIFENAS, parecer \#1.629.220. O Termo de Consentimento Livre e Esclarecido (TCLE) foi assinado pelos pais ou responsável legal dos pacientes com FLP.

\section{Resultados}

Conforme apresentado na Tabela 1, a maioria dos pacientes com FLP foram do sexo masculino (62,38\%), declarados leucoderma $(82,67 \%)$, e pertencentes as classes socioeconômicas de mais baixa renda. Quanto ao tipo de trabalho da mãe, houve um equilíbrio entre a área urbana $(35,14 \%)$, área rural $(33,16 \%)$, e as que não trabalham $(31,68 \%)$. Dentre aquelas com trabalho na área rural $(n=67), 46,27 \%$ estavam em contato com algum tipo de agrotóxico/herbicida. A maioria dos pais não informaram emprego, e $26,23 \%$ trabalhavam na área rural, sendo que 34 estavam em contato com agrotóxico/herbicida. 
Tabela 1 - Perfil sociodemográfico dos 202 pacientes $(n=202)$ na faixa etária entre 1 e 10 anos, atendidos em um centro de referência no atendimento de pacientes com fissuras labiopalatais. Alfenas/MG, Brasil, 2016-2017.

\begin{tabular}{|c|c|c|c|}
\hline VARIÁVEL & $n$ & $\%$ & IC95\% \\
\hline \multicolumn{4}{|l|}{ Sexo } \\
\hline Masculino & 126 & 62,38 & $55,7-69,1$ \\
\hline Feminino & 76 & 37,62 & $30,9-44,3$ \\
\hline \multicolumn{4}{|l|}{ Raça } \\
\hline Leucoderma & 167 & 82,67 & $77,5-87,9$ \\
\hline Feoderma/Melanoderma & 26 & 12,87 & $8,3-17,5$ \\
\hline Xantoderma & 9 & 4,46 & $1,6-7,3$ \\
\hline \multicolumn{4}{|l|}{ Classe social } \\
\hline A1 & 0 & 0 & ---- \\
\hline A2 & 2 & 0,99 & ---- \\
\hline B1 & 3 & 1,5 & ---- \\
\hline B2 & 25 & 12,37 & $7,8-16,9$ \\
\hline $\mathrm{C}$ & 136 & 67,32 & $60,9-73,8$ \\
\hline $\mathrm{D}$ & 34 & 16,83 & $11,7-22$ \\
\hline E & 2 & 0,99 & ---- \\
\hline \multicolumn{4}{|l|}{ Local de trabalho da mãe } \\
\hline Área urbana & 71 & 35,14 & $28,6-41,7$ \\
\hline${ }^{1}$ Área rural & 67 & 33,16 & $26,7-39,7$ \\
\hline Não trabalha & 64 & 31,68 & $25,3-38,1$ \\
\hline \multicolumn{4}{|l|}{ 1Área rural: contato com agrotóxico } \\
\hline Sim & 31 & 46,27 & $34,3-58,2$ \\
\hline Não & 4 & 5,97 & ---- \\
\hline Não soube informar & 32 & 47,76 & $35,8-59,7$ \\
\hline \multicolumn{4}{|l|}{ Local de trabalho do pai } \\
\hline Área urbana & 23 & 11,38 & $7-15,8$ \\
\hline${ }^{2}$ Área rural & 53 & 26,23 & $20,2-32,3$ \\
\hline Não informou & 126 & 62,37 & $55,7-69,1$ \\
\hline \multicolumn{4}{|l|}{${ }^{2}$ Área rural: contato com agrotóxico } \\
\hline Sim & 34 & 64,15 & $51,2-77,1$ \\
\hline Não & 4 & 7,55 & ---- \\
\hline Não soube informar & 15 & 28,3 & $16,2-40,4$ \\
\hline
\end{tabular}

Quanto aos dados clínicos, conforme apresentado na Tabela 2, os tipos mais frequentes de FLP foram: pré-forame incompleto $(13,36 \%, \mathrm{n}=27)$, transforame completo esquerda $(13,36 \%, \mathrm{n}=27)$, pré-forame completo $(10,89 \%, \mathrm{n}=22)$, transforame completo bilateral $(6,43 \%, \mathrm{n}=13)$ e transforame direito $(11,38 \%, \mathrm{n}=23)$.

Com relação ao grupo sanguíneo (Tabela 2), a maioria pertence ao grupo O positivo $(35,65 \%)$, seguido de A positivo $(17,82 \%)$ e B positivo $(5,45 \%)$. Os procedimentos cirúrgicos mais frequentes foram: queiloplastia (48,81\%), palatoplastia $(38,97 \%)$, rinoplastia (9,84\%), labioplastia (2,36\%). 20,29\% dos entrevistados ainda não passaram por procedimento cirúrgico.

Ainda conforme apresentado na Tabela 2, entre os pacientes com FLP, 55,44\% tiveram peso ao nascer entre 3.000 e 4.000 gramas, $35,15 \%$ entre 2.000 e 3.000 gramas, $3,47 \%$ entre 1.000 e 2.000 gramas, $0,50 \%$ entre 5.000 e 6.000 gramas. A maioria dos pacientes com FLP $(53,46 \%)$ apresentou alguma comorbidade, sendo, as mais comuns: respiratória $(51,85 \%)$, alérgica $(32,40 \%)$, anemia $(24,07 \%)$, gástrica $(14,81 \%)$ e cardíaca $(12,03 \%)$. O acompanhamento com fonoaudiólogo foi relatado por 73,76\% dos entrevistados. 
Tabela 2 - Perfil clínico dos 202 pacientes $(\mathrm{n}=202)$, atendidos em um centro de referência no atendimento de pacientes com fissuras labiopalatais. Alfenas/MG, Brasil, 2016-2017.

\begin{tabular}{|c|c|c|c|}
\hline VARIÁVEL & $n$ & $\%$ & IC95\% \\
\hline \multicolumn{4}{|l|}{ Tipo de fissura labiopalatal } \\
\hline Forame bilateral incompleto & 4 & 1,98 & ---- \\
\hline Forame bilateral completo & 13 & 6,43 & $3,1-9,8$ \\
\hline Forame unilateral labial completo & 6 & 2,97 & $0,6-5,3$ \\
\hline Forame labial esquerdo & 10 & 4,95 & $2-7,9$ \\
\hline Forame labial direito & 6 & 2,97 & $0,6-5,3$ \\
\hline Pós forame incompleto & 32 & 15,84 & $10,8-20,9$ \\
\hline Pós forame completo bilateral & 9 & 4,45 & $1,6-7,3$ \\
\hline Pré forame completo & 22 & 10,89 & $6,6-15,2$ \\
\hline Pré forame incompleto & 27 & 13,36 & $8,7-18,1$ \\
\hline Transforame completo bilateral & 13 & 6,43 & $3,1-9,8$ \\
\hline Transforame completo esquerda & 27 & 13,36 & $8,7-18,1$ \\
\hline Transforame direito & 23 & 11,38 & $7-15,8$ \\
\hline Transforame incompleto & 5 & 2,47 & --- \\
\hline Fenda submucosa & 2 & 0,99 & ---- \\
\hline Não foi descrito & 3 & 1,48 & ---- \\
\hline \multicolumn{4}{|l|}{ Peso ao nascer (gramas) } \\
\hline 1.000 a 2.000 & 7 & 3,47 & $0,9-6$ \\
\hline 2.000 a 3.000 & 71 & 35,14 & $28,6-41,7$ \\
\hline 3.000 a 4.000 & 112 & 55,44 & $48,6-62,3$ \\
\hline 5.000 a 6.000 & 1 & 0,50 & ---- \\
\hline Não informado & 11 & 5,44 & $2,3-8,6$ \\
\hline \multicolumn{4}{|l|}{ Tipo sanguíneo } \\
\hline $\mathrm{A}+$ & 36 & 17,82 & $12,5-23,1$ \\
\hline $\mathrm{B}+$ & 11 & 5,44 & $2,3-8,6$ \\
\hline $\mathrm{AB}+$ & 2 & 0,99 & --- \\
\hline $\mathrm{O}+$ & 72 & 35,65 & $29-42,2$ \\
\hline A- & 6 & 2,97 & $0,6-5,3$ \\
\hline B- & 0 & 0 & --- \\
\hline AB- & 0 & 0 & ---- \\
\hline O- & 4 & 1,98 & ---- \\
\hline Não informado & 71 & 35,14 & $28,6-41,7$ \\
\hline \multicolumn{4}{|l|}{ Procedimento cirúrgico realizado* } \\
\hline Queiloplastia & 99 & 48,81 & $42-56$ \\
\hline Palatoplastia & 79 & 38,97 & $32,4-45,8$ \\
\hline Rinoplastia & 20 & 9,84 & $5,8-14$ \\
\hline Labioplastia & 5 & 2,36 & --- \\
\hline Não foi submetido à cirurgia & 41 & 20,29 & $14,8-25,8$ \\
\hline \multicolumn{4}{|l|}{ Acompanhamento com Fonoaudiólogo } \\
\hline Sim & 149 & 73,76 & $67,7-79,8$ \\
\hline Não & 42 & 20,8 & $15,2-26,4$ \\
\hline Não informado & 11 & 5,44 & $2,3-8,6$ \\
\hline \multicolumn{4}{|l|}{ Sofre de alguma comorbidade ${ }^{* *}$} \\
\hline $\operatorname{Sim}^{\mathrm{A}}$ & 108 & 53,46 & $47-60$ \\
\hline Não & 94 & 46,53 & $39,7-53,4$ \\
\hline \multicolumn{4}{|l|}{${ }^{A}$ Comorbidades } \\
\hline Respiratória & 56 & 51,85 & $42,4-61,3$ \\
\hline Cardíaca & 13 & 12,03 & $5,9-18,2$ \\
\hline Alérgica & 35 & 32,40 & $23,6-41,2$ \\
\hline Gástrica & 16 & 14,81 & $8,1-21,5$ \\
\hline Urogenital & 8 & 7,40 & $2,5-12,3$ \\
\hline Anemia & 26 & 24,07 & $16-32,1$ \\
\hline Autismo & 1 & 0,92 & --- \\
\hline Epilepsia & 1 & 0,92 & ---- \\
\hline Paralisia & 1 & 0,92 & ---- \\
\hline
\end{tabular}

* Foram 254 cirurgias realizadas, visto que alguns pacientes foram submetidos a mais de um tipo de cirurgia.

** O mesmo paciente pode apresentar mais de um tipo de comorbidade. 
Com relação aos dados familiares, conforme apresentado na Tabela 3, 30,7\% dos pacientes com FLP entrevistados possuem antecedentes familiares para as FLP. Observou-se também que, uma grande parcela das mães entrevistadas, 70,8\%, fazia uso da suplementação com ácido fólico durante a gestação.

Tabela 3 - Dados referentes aos antecedentes gestacional e familiar na amostra de 202 pacientes $(n=202)$ atendidos em um centro de referência no atendimento de pacientes com fissuras labiopalatais. Alfenas/MG, Brasil, 2016-2017.

\begin{tabular}{|c|c|c|c|}
\hline VARIÁVEL & $n$ & $\%$ & IC95\% \\
\hline \multicolumn{4}{|l|}{$\begin{array}{l}\text { MÃES } \\
\text { Uso de ácido fólico durante a gestação }\end{array}$} \\
\hline  & 143 & 70,8 & $64,5-77,1$ \\
\hline Não & 49 & 24,25 & $18,3-30,2$ \\
\hline Não informado & 10 & 4,95 & $2-7,9$ \\
\hline \multicolumn{4}{|l|}{$\begin{array}{l}\text { HISTÓRICO FAMILIAR } \\
\text { Antecedentes de alterações craniofaciais } \\
\text { congênita }\end{array}$} \\
\hline Sim & 62 & 30,7 & $24,3-37,1$ \\
\hline Não & 140 & 69,3 & $62,9-75,7$ \\
\hline
\end{tabular}

\section{Discussão}

Este estudo analisou uma amostra de 202 pacientes com FLP, na faixa etária entre $1 \mathrm{e}$ 10 anos, assistidos em um centro de referência do Sul de Minas Gerais. Existe diferenças entre as raças e o sexo quanto a incidência das FLP. Freitas \& Silva et al. observaram uma maior frequência de FLP com ou sem envolvimento do palato para o sexo masculino e predominância do sexo feminino apenas para os casos de FLP isolada (7). Shibukawa et al. (3), entre 2005 a 2016, analisando o registro de 17.800 nascidos vivos brasileiros com FLP, também observaram predominância de leucodermas e do sexo masculino. Assim, é descrito que leucodermas e o sexo masculino são mais acometidos pela FLP $(3,7,9)$, sendo que estes prévios achados estão em consonância com o presente estudo.

O nível socioeconômico é um fator preponderante quando se avalia a ocorrência das FLP, uma vez que, nas classes sociais menos favorecidas, a ocorrência dessas alterações são mais comuns $(2,5,7)$, como também observado neste estudo, no qual a maior parte dos entrevistados pertencia às classes B2, C e D. Neste contexto, influenciando sobremaneira a gênese das FLP bem como a promoção de saúde e qualidade de vida das pessoas vivendo com esta doença, a incidência aumentada nestas classes pode estar relacionada a fatores das gestantes, incluindo os nutricionais, ocupacionais, acesso à informação e serviços de saúde, bem como a triagem e diagnóstico neonatal $(3,9)$. Também houve uma melhoria contínua nas notificações e, consequentemente, o registro de um número de casos mais próximo do real $(3,10,11)$.

Em termos clínicos, as FLP são resultantes de uma falha na fusão do lábio e/ou palato, em que a formação da face representa um dos complexos eventos do desenvolvimento embrionário humano, assim esta doença pode apresentar-se sob diversas formas clínicas $(3,12,13)$. Assim, relatos apontam que as FLP transforames são as mais prevalentes, com uma razão entre os sexos de aproximadamente 1,5 masculino:1,0 feminino $(2,5)$. Estes prévios achados são semelhantes aos apresentados no presente estudo.

Neste estudo, a queiloplastia e a palatoplastia foram as cirurgias eleitas mais frequentes, e uma considerável parcela dos entrevistados ainda não forma submetidos a cirurgia. Estes achados são semelhantes aos encontrados por da Silva et al. que observou pacientes com FLP em Natal, RN, Brasil (9). Neste mesmo sentido, por 
afetarem funções que interferem no desenvolvimento psicológico, fisiológico e na adaptação social, além da cirurgia, as FLP requerem outros tipos de cuidados, visando melhorias na qualidade de vida e promoção de saúde dos pacientes vivendo com esta doença $(9,12,14)$. Neste contexto, a atuação multiprofissional é fundamental $(7,13)$ e, como observado no presente estudo, a maior parte dos pacientes estava em acompanhamento com Fonoaudiólogo.

No presente estudo, com relação aos antecedentes familiares, uma considerável parcela dos entrevistados relatou ter algum familiar de primeiro grau (mãe e/ou pai e/ou irmã e/ou filha) com alteração orofacial congênita. De acordo com a literatura, a presença de um ou mais familiar de primeiro grau com alteração orofacial congênita eleva o risco de recorrência em probandos (3,15-18). Este risco de recorrência pode saltar de $<1 \%$ para $4 \%$ quando a gestante apresenta um familiar de primeiro grau com FLP, e para $10 \%$ quando há dois $(15,17)$.

Por se tratar de uma doença Genética multifatorial, fatores ambientais podem precipitar o aparecimento das FLP quando há uma predisposição hereditária (4,19-21). Portanto, além dos fatores genéticos/hereditários na gênese das FLP, diversos fatores ambientais são considerados de risco para o desenvolvimento desta doença. Dentre estes, a deficiência de ácido fólico entre gestantes tem sido reportada como um risco materno para as FLP (5). Como observado no presente estudo, a maioria das gestantes fez uso de suplementação com ácido fólico durante a gestação, contrastando com os resultados encontrados por da Silva et al. (9). Ainda, a maior parte dos pacientes com FLP apresentou peso normal ao nascimento (>2.500 gramas) (3), com maior frequência de nascidos com peso entre 3.000 e 4.000 gramas, semelhante aos achados de Silva et al. (9) e Shibukawa et al. (3). Além do efeito protetor contra os riscos de surgimento das FLP, reportados em literatura (5), na amostra de pacientes avaliada no presente estudo, a suplementação com ácido fólico pela maior parcela das gestantes pode destacar a importância dos cuidados durante a gestação, contribuindo com uma gestação mais saudável e para a saúde do neonato com FLP (como ótimo peso ao nascer).

A exposição ocupacional durante a gestação também se caracteriza como um sério fator de risco para as alterações congênitas, sendo os agrotóxicos/herbicidas conhecidos teratógenos e, estes agentes químicos têm sido associados a gênese das FLP (22-25). Como visto no presente estudo, grande parte dos pais dos pacientes vivendo com FLP, trabalhavam na zona rural, com um potencial para a exposição ocupacional aos agrotóxicos/herbicidas.

De acordo com da Silva et al. (9), a anemia, as alterações respiratórias e cardiovasculares foram as três comorbidades mais frequentes, estes resultados são semelhantes aos encontrados no presente estudo, o que mostra que pacientes com FLP são mais susceptíveis a tais comorbidades. Além disso, pacientes com FLP também apresentam outras alterações maiores ou menores, sendo a doença congênita do coração e a Sequência de Pierre Robin as mais comuns (26).

\section{Conclusão}

Este estudo permite concluir que para a amostra analisada, de pacientes entre 1 a 10 anos de idade com FLP, houve predominância de leucodermas e do sexo masculino, as fissuras pós-forame incompleto, pré-forame incompleto e transforame completa esquerda foram as mais comuns, e os tratamentos cirúrgicos mais frequentes foram a queiloplastia e a palatoplastia. Acompanhamento com fonoaudiólogo foi relatado pela maioria. A maior parte das mães dos pacientes estava sob suplementação com ácido fólico durante a gestação, e o peso ao nascimento foi normal para a maioria dos 
pacientes. Os pacientes que apresentaram comorbidades foram mais acometidos por doenças respiratórias, alergias e anemia.

Além disso, a frequência de pacientes com histórico familiar para algum tipo de alteração craniofacial congênita, associado a potencial exposição ocupacional dos pais, bem como o fato das classes econômicas menos favorecidas serem as mais afetadas, reforçam a plausível etiologia multifatorial das FLP. Portanto, fatores sociais e clínicos interferem na promoção de saúde para pacientes com FLP, sendo necessário atenção as gestantes e acompanhamento multiprofissional constante dos pacientes. Desse modo, é importante a manutenção além da implementação de melhorias nas políticas públicas de saúde, do SUS, considerando os achados deste estudo, que corroboram o fato da FLP acometer as classes mais vulneráveis sócio e economicamente.

\section{Agradecimentos}

Os autores agradecem aos pacientes voluntários, familiares e profissionais do Centro Pró Sorriso da Unifenas.

\section{Referências}

1. Cosme HW, Lima LS, Barbosa LG, Prevalência de anomalias congênitas e fatores associados em recém-nascidos do município de São Paulo no período de 2010 a 2014. Rev Paul Pediatr 2017; 35(1): $33-8$.

2. Kosowski TR, Weathers WM, Wolfswinkel EM, Ridgway EB. Cleft Palate. Seminars in Plastic Surgery 2012; 26(4): 164-169.

3. Shibukawa BMC, Rissi GP, Higarashi IH, et al. Fatores associados à presença de fissura labial e/ou fenda palatina em recém nascidos brasileiros. Rev Bras Saúde Mater Infant. 2019; 19 (4): 957-966.

4. Kohli SS, Kohli VL. A comprehensive review of the genetic basis of cleft lip and palate. J Oral Maxillofac Pathol 2012; 16(1): 64-72.

5. Shkoukani MA, Chen M, Vong A. Cleft lip - a comprehensive review. Frontiers in Pediatrics 2013; 1(53): 10 .

6. Antoniazzi TF, Coto NP, Pereira SL, Cardim RH, Dias RB. Etiologia das fissuras de lábio e/ou palato: estudo estatístico. Rev Odontol Univ Santo Amaro 2005; 10(1): 20-3.

7. Freitas e Silva DA, Mauro LDL, Oliveira LB, Ardenghi TM, Bonecker M. Estudo descritivo de fissuras lábio-palatinas relacionadas a fatores individuais, sistêmicos e sociais. RGO 2008; 56(4): 387-391.

8. Spina V, Psillakis JM, Lapa FS. Classificação das fissuras lábio-palatais. Sugestão de modificação. Rev Hosp Clin Fac Med 1972; (27): 5-6.

9. da Silva HPV, Arruda TTS, de Souza KSC, et al. Risk factors and comorbidities in Brazilian patients with orofacial clefts. Braz Oral Res. 2018; 32: e24.

10. Loffredo LCM, Freitas JAS, Grigolli AAG. Prevalência de fissuras orais de 1975 a 1994. Rev Saúde Pública 2001; 35(6): 571-5.

11. Abreu MHNG, Lee KH, Luquetti DV, Starr JR. Temporal trend in the reported birth prevalence of cleft lip and/or cleft palate in Brazil, 2000 to 2013. Birth Defects Res Part A Clin Mol Teratol 2016; 106 (9): 789-92.

12. Sousa GFT, Roncalli AG. Orofacial clefts in Brazil and surgical rehabilitation under the Brazilian National Health System. Braz Oral Res 2017; 31: e23.

13. Melgaço CA, Di Ninno CQMS, Penna LM, do Vale MPP. Aspecto ortodôntico/ortopédicos e fonoaudiológicos relacionados a pacientes portadores de fissuras labiopalatinas. J Bras Ortodon Ortop Facial 2002; 7(37): 23-32.

14. Montagnoli LC, Barbieri MA, Bettiol H, Marques IL, Souza L. Prejuízo no crescimento de crianças com diferentes tipos de fissura lábio-palatina nos 2 primeiros anos de idade: um estudo transversal. Jornal de Pediatria 2005; 81(6): 461- 465. 
15. Klotz CM, Wang X, Desensi RS, Grubs RE, Costello BJ, Marazita ML. Revisiting the Recurrence Risk of Nonsyndromic Cleft Lip with or without Cleft Palate. Am J Med Genet A 2010; 152A(11): 2697-2702.

16. Funato N, Nakamura M. Identification of shared and unique gene families associated with oral clefts. International Journal of Oral Science 2017; 9: 104-109.

17. Grosen D, Chevrier C, Skytthe A, Bille C, Mølsted K, Sivertsen A, Murray JC, Christensen K. A cohort study of recurrence patterns among more than 54000 relatives of oral cleft cases in Denmark: support for the multifactorial threshold model of inheritance. J Med Genet 2010; 47: 162e168.

18. Sivertsen A, Wilcox AJ, Skjaerven R, Vindenes HA, Abyholm F, Harville E, Lie RT. Familial risk of oral clefts by morphological type and severity: population based cohort study of first degree relatives. BMJ 2008; 336(7641): 432-4.

19. Saleem K, Zaib T, Sun W, Fu S. Assessment of candidate genes and genetic heterogeneity in human non syndromic orofacial clefts specifically non syndromic cleft lip with or without palate. Heliyon. 2019; 5: e03019.

20. Beaty TH, Marazita ML, Leslie EJ. Genetic factors influencing risk to orofacial clefts: today's challenges and tomorrow's opportunities. F1000Research 2016; 5: 2800.

21. Leslie EJ, Marazita ML. Genetics of Cleft Lip and Cleft Palate. Am J Med Genet C Semin Med Genet 2013; 163(4): 246-258.

22. Spinder N, Bergman JEH, Boezen HM, Vermeulen RCH, Kromhout H, de Walle HEK. Maternal occupational exposure and oral clefts in offspring. Environmental Health 2017; 16: 83.

23. Suhl J, Romitti PA, Rocheleau C, Cao Y, Burns TL, Conway K, Bell EM, Stewart P, Langlois P; National Birth Defects Prevention Study. Parental occupational pesticide exposure and nonsyndromic orofacial clefts. J Occup Environ Hyg 2018; 15(9): 641-653.

24. Leite ICG, Paumgartten FJR, Koifman S. Fendas orofaciais no recém-nascido e exposições ambientais e ocupacionais dos pais: um estudo caso-controle no Rio de Janeiro, Brasil. Rev Bras Saúde Matern Infant 2003; 3(4): 401-409.

25. Leite ICG, Paumgartten FJR, Koifman S. Exposição a agentes químicos na gravidez e fendas lábiopalatinas no recém-nascido. Cad Saúde Pública 2002; 18(1): 17-31.

26. Varandas ET, Regis LAF, Furtado PGC, Guimarães SMF. Malformações congênitas associadas às fissuras lábio palatinas: análise de 22 casos. Rev Odontol Univ Santo Amaro 1997; 2(3): 15-8. 